Full length article

\title{
Can radical surgical treatment of the vulva be justified in the absence of a conclusive diagnosis of squamous cell carcinoma on biopsy? A retrospective 10 -year cohort study
}

\author{
Lysanne W. Jonker ${ }^{\mathrm{a}, 1, *}$, Shatavisha Dasgupta ${ }^{\mathrm{b}, 1}$, Patricia C. Ewing-Graham ${ }^{\mathrm{b}}$, \\ Helena C. van Doorn ${ }^{\mathrm{a}}$ \\ a Department of Gynecologic Oncology, Erasmus MC Cancer Institute, Rotterdam, the Netherlands \\ ${ }^{\mathrm{b}}$ Department of Pathology, Erasmus MC, University Medical Centre Rotterdam, the Netherland
}

\section{A R T I C L E I N F O}

\section{Article history:}

Received 20 December 2019

Received in revised form 9 March 2020

Accepted 15 March 2020

Available online $\mathrm{xxx}$

\section{Keywords:}

Vulva

Vulvar neoplasms

Squamous cell carcinoma

Gynecologic surgical procedures

Vulvectomy

\begin{abstract}
A B S T R A C T
Objectives: The extent of surgical treatment for vulvar lesions is predominantly guided by the histopathologic diagnosis rendered on the pre-operative biopsy. For premalignant lesions, local excisions are performed, whereas for vulvar squamous cell carcinoma (VSCC), more radical procedures are mandatory. However, even in the absence of a conclusive diagnosis of VSCC on biopsy, the surgeon may opt for a radical excision on grounds of strong clinical suspicion, with a view to avoiding repeat surgeries. We studied a retrospective, 10-year cohort of patients who underwent vulvar excisions, in the absence of a conclusive biopsy diagnosis of VSCC. We aimed to identify the factors predictive of VSCC in these patients, and assess their treatment.

Study design: All patients who underwent vulvar excision (2005-2016) at Erasmus MC, without a definitive diagnosis of VSCC on the preoperative biopsy were included. Logistic regression analysis was performed to identify the factors predictive of a final diagnosis of VSCC. Surgical treatment was categorized as definitive, incomplete, or over-treatment, based on histopathology of the excision specimen and previous surgical history.

Results: In 57 \% (64/113) of all included patients, the final diagnosis was VSCC. Higher patient age ( $\mathrm{p}=$ 0.03 ), and suspicion of VSCC on pre-operative biopsy ( $p<0.001)$ were associated with a final diagnosis of VSCC on univariate analysis. Suspicion of VSCC on biopsy was the only significant predictor $(\mathrm{p}<0.001)$ on multivariable analysis. For patients with a suspicion of VSCC on biopsy, radical treatment was more frequently performed $(\mathrm{p}<0.001)$, which resulted in over-treatment in only 1 case. Where the surgeon had performed a limited excision despite a suspicion of VSCC on biopsy, high patient age, co-morbidities, location of the tumor close to the anus, and history of previous vulvar surgeries were factors which influenced the decision. The treatment administered was definitive for $72 \%$., i.e. additional surgeries were not required; $25 \%$ received incomplete treatment and needed additional surgeries, and $3 \%$ received over-treatment.

Conclusion: Suspicion of VSCC on biopsy is strongly predictive of a final diagnosis of carcinoma. In our cohort, radical treatment performed on patients with clinical and histopathological suspicion of VSCC resulted in minimal over-treatment, and helped avoid second surgeries.
\end{abstract}

(c) 2020 Elsevier B.V. All rights reserved.

\footnotetext{
* Corresponding author at: Department of Radiation Oncology, Amsterdam UMC, Vrije Universiteit Amsterdam, de Boelelaan 1117, 1081HV, Amsterdam, the Netherlands.

E-mail addresses: l.jonker1@amsterdamumc.nl (L.W. Jonker), s.dasgupta@erasmusmc.nl (S. Dasgupta),p.ewing@erasmusmc.nl (P.C. Ewing-Graham), h.vandoorn@erasmusmc.nl (H.C. van Doorn).

${ }^{1}$ These authors contributed equally to the article, and share first authorship.
}

\section{Introduction}

Vulvar lesions clinically suspected to be vulvar squamous cell carcinoma (VSCC), or its precursor, vulvar intraepithelial neoplasia (VIN), are biopsied by the surgeon, and the subsequent management of the patient is guided by the histopathologic diagnosis on the biopsy. For VIN, patients receive topical treatment, ablative therapy, or limited local excision [1-5]. For VSCC, more extensive excisions are performed, if necessary in combination with groin 
treatment, i.e. sentinel lymph node (SLN) procedure, or groin lymph node dissection (LND) [6,7].

The diagnosis of VSCC may however be missed on the preoperative biopsy in $8-22 \%$ of cases [8-11].This is often due to sampling error, or in some cases, due to improper orientation or sectioning of the biopsy specimen, which hinder adequate histopathologic assessment. When VSCC is not conclusively diagnosed on the pre-operative biopsy, but the clinical suspicion is sufficiently high, the surgeon may opt to perform a radical excision, with or without groin treatment. Quite often in these cases, VSCC is diagnosed in the excision specimen, and the surgeon manages to spare the patient a repeat surgery in the anatomically complex vulvar region. However, if the excision specimen does not show VSCC, it implies that the patient was over-treated. For these cases, an insight into the specific features that may predict the presence of VSCC could improve clinical decision-making.

This exploratory study was therefore conducted on a cohort of patients who underwent surgical treatment of the vulva, in the absence of a conclusive diagnosis of VSCC on pre-operative biopsy. The aims were to identify the factors that might predict the presence of VSCC, and to assess the surgical treatments performed.

\section{Materials and methods}

This retrospective, single-center, cohort-study was conducted at Erasmus MC Cancer Institute, which is a tertiary referral center for gynecologic malignancies. Approval of the Medical Research Ethics Committee was obtained (MEC 2017-134).

\section{Patient population}

All patients who underwent vulvar surgical excisions between January 1, 2005, and January 1, 2016 were identified from the records of the Department of Gynecologic Oncology. Of these, patients who did not have a conclusive diagnosis of VSCC on their pre-operative biopsy(ies) were included. All patient data were anonymized.

If the patient underwent vulvar biopsy followed by an excision more than once, only the first episode was analyzed. Patients with vulvar malignancies other than conventional VSCC, e.g. verrucous carcinoma, basal cell carcinoma, adenocarcinoma, sarcoma, or melanoma, were excluded.

\section{Data retrieval}

Clinical data such as age, diameter and focality of the lesion as noted during clinical examination, history of previous vulvar lesions, treatment details of previous, current, and subsequent episodes, and follow-up, were collected from patient records. Remarks on the treatment rationale, where available, were also recorded.

Pathology data were extracted from the reports of the preoperative biopsy and the post-operative excision specimens. For VIN, the histopathologic type, i.e. differentiated VIN (dVIN), or high grade squamous intraepithelial lesion (HSIL) was recorded. For VSCC, tumor size, depth of invasion, focality, differentiation grade, and presence of perineural or lymphovascular space invasion were recorded. Status of SLN or LND specimens was noted as positive or negative for metastasis. Slides from all external referral cases had been reviewed by the departmental pathologists before the surgical procedure. All cases where the slides were still available in the departmental archive were reviewed by two pathologists (SDG and PEG) for the purpose of this study. PEG is an experienced gynecologic pathologist.

\section{Categorization of surgical treatments}

The surgical procedures were categorized as:

\section{Local treatment}

Wide local excision (WLE), or radical vulvectomy (RV).

The aim was to excise with a margin of $5 \mathrm{~mm}$ for VIN, and 10 $\mathrm{mm}$ for VSCC, but this was not always achievable.

\section{Groin treatment \\ SLN procedure, or full groin LND.}

\section{Radical treatment}

Treatment of both vulva and groin(s), i.e. WLE or RV, with groin treatment.

For each patient, the treatment was categorized as definitive, incomplete, or over-treatment, based on the histopathologic diagnosis of the post-operative specimen, and taking into account the previous history of vulvar surgery, as elaborated below.

\section{Definitive treatment}

The surgical procedure was deemed sufficient, and no additional procedures were required, e.g. WLE for VIN, or radical treatment for primary VSCC. For recurrent VSCC, where groin treatment had been conducted previously, WLE with adequate margins was considered definitive.

\section{Incomplete treatment}

The surgical procedure was deemed insufficient, and additional procedures were required, e.g. where WLE had been performed, and VSCC stage T1b was diagnosed in the post-operative excision specimen; this meant a subsequent SLN procedure was needed.

\section{Over-treatment}

The surgical treatment was deemed to be in excess of what was necessary, e.g. where radical treatment had been performed, and the post-operative excision specimen did not show VSCC.

\section{Statistical analyses}

Data were analyzed using SPSS Statistics 25 (IBM Corp., Armonk, NY, USA). Descriptive statistic was used for patient characteristics. Independent sample's $t$-test was used for continuous data, and Chi-squared $\left(\chi^{2}\right)$ test for categorical data. Logistic regression analysis was performed to study the effect of several variables on the final diagnosis of VSCC, which was considered as a dichotomous outcome (present or absent). Subgroup analysis of patients with or without a suspicion of VSCC on pre-operative biopsy was also conducted. Two-sided p-value $<0.05$ was considered significant.

\section{Results}

Of 1245 patients who underwent vulvar excisions, 113 met the inclusion criteria.

\section{Characteristics of all included patients}

The patients had a mean age of 65.3 years (range 24-91 years). The median lesional diameter was $21.5 \mathrm{~mm}$, and the lesion was unifocal in $77 \%(87 / 113)$ of patients [Table 1 ].

Suspicion of VSCC was present on the pre-operative biopsy in $49 \%(55 / 113)$ of patients [Table 1 ]. Local treatment was performed for $75 \%(85 / 113)$, and radical treatment for $25 \%(28 / 113)$ of patients. The final diagnosis on post-operative histopathology was VSCC for $57 \%$ (64/113), dVIN for $13 \%$ (15/113), HSIL for $29 \%$ (33/ 
Table 1

Patient characteristics.

\begin{tabular}{|c|c|c|c|c|}
\hline & All $(n=113)$ & With VSCC $(n=64)$ & Without VSCC $(n=49)$ & p-value* \\
\hline \multicolumn{5}{|l|}{ I. Age (in years) } \\
\hline Mean (95\% Confidence Interval) & $65.3(62.3-68)$ & $67.9(64.8-71.2)$ & $61.2(57.1-66.4)$ & 0.03 \\
\hline \multirow[t]{2}{*}{ Range } & $24-91$ & $32-88$ & 24-91 & \\
\hline & \multicolumn{3}{|c|}{ Number of patients (percentage) } & \\
\hline \multicolumn{5}{|l|}{ II. History of vulvar lesions } \\
\hline None & $65(58)$ & $39(61)$ & $26(53)$ & 0.60 \\
\hline Lichenoid lesions & $9(8)$ & $6(9)$ & $3(6)$ & \\
\hline VIN & $17(15)$ & $9(14)$ & $8(16)$ & \\
\hline VSCC & $22(19)$ & $10(16)$ & $12(25)$ & \\
\hline III. Previous groin treatment & $19(17)$ & $9(14)$ & $10(20)$ & 0.37 \\
\hline IV. Previous radiotherapy & $6(5)$ & $5(7)$ & $1(2)$ & 0.18 \\
\hline \multicolumn{5}{|l|}{ V. Preoperative clinical examination } \\
\hline \multicolumn{5}{|l|}{ A. Diameter of the lesions } \\
\hline Median (Range) in mm & $21.5(3-70)$ & $25(3-65)$ & $20(3-70)$ & 0.75 \\
\hline$<20 \mathrm{~mm}$ & $41(36)$ & $25(39)$ & $16(33)$ & \\
\hline$\geq 20 \mathrm{~mm}$ & $71(63)$ & $38(59)$ & $33(67)$ & 0.51 \\
\hline Unknown & $1(1)$ & $1(2)$ & $0(0)$ & \\
\hline \multicolumn{5}{|l|}{ B. Focality } \\
\hline Unifocal & $87(77)$ & $49(77)$ & $38(78)$ & 1.00 \\
\hline Multifocal & $26(23)$ & $15(23)$ & $11(22)$ & \\
\hline VI. Preoperative histopathology & & & & $<0.001$ \\
\hline No suspicion of VSCC & $58(51)$ & $19(30)$ & $39(80)$ & \\
\hline Suspicion of VSCC & $55(49)$ & $45(70)$ & $10(20)$ & \\
\hline VII. Surgical procedure & & & & $<0.001$ \\
\hline \multicolumn{5}{|l|}{ A. Local treatment } \\
\hline WLE & $85(75)$ & $40(63)$ & $45(92)$ & \\
\hline \multicolumn{5}{|l|}{ B. Radical treatment } \\
\hline WLE + SLN procedure & $19(17)$ & $15(23)$ & $4(8)$ & \\
\hline WLE + LND & $9(8)$ & $9(14)$ & $0(0)$ & \\
\hline VIII. Treatment categorization & & & & $<0.01$ \\
\hline Definitive & $81(72)$ & $36(56)$ & $45(92)$ & \\
\hline Incomplete treatment & $28(25)$ & $28(44)$ & $0(0)$ & \\
\hline Over-treatment & $4(3)$ & $0(0)$ & $4(8)$ & \\
\hline IX. Additional treatment & & & & 0.0001 \\
\hline None & $82(73)$ & $33(52)$ & $49(100)$ & \\
\hline Local treatment (WLE) & $6(5)$ & $6(9)$ & $0(0)$ & \\
\hline Radical treatment (WLE + groin treatment) & $11(10)$ & $11(17)$ & $0(0)$ & \\
\hline Groin treatment only & $11(10)$ & $11(17)$ & $0(0)$ & \\
\hline Radiotherapy & $3(2)$ & $3(5)$ & $0(0)$ & \\
\hline X. Follow up & \multicolumn{4}{|c|}{ Median follow-up duration: 24 months } \\
\hline No evidence of disease & $90(80)$ & $48(75)$ & $42(86)$ & \\
\hline \multicolumn{5}{|l|}{ Death } \\
\hline Related to disease & $3(2)$ & $3(5)$ & $0(0)$ & \\
\hline Unrelated to disease & $4(3)$ & $3(5)$ & $1(2)$ & \\
\hline \multicolumn{5}{|l|}{ Recurrence of disease } \\
\hline VIN & $7(7)$ & $3(5)$ & $4(8)$ & \\
\hline VSCC & $3(2)$ & $2(3)$ & $1(2)$ & \\
\hline Under palliative care & $5(5)$ & $5(7)$ & $0(0)$ & \\
\hline No follow up & $1(1)$ & $0(0)$ & $1(2)$ & \\
\hline
\end{tabular}

VIN: vulvar intraepithelial neoplasia, VSCC: vulvar squamous cell carcinoma, WLE: wide local excision, SLN: sentinel lymph node, LND: lymph node dissection. Univariate analysis.

113), and lichenoid changes for $1 \%(1 / 113)$ of patients. Pathology details of the VSCCs are presented in Table 2.

The surgical procedure performed was definitive for $72 \%$ ( $81 /$ $113)$, incomplete for $25 \%$ (28/113), and over-treatment for $3 \%$ (4/ 113 ) of patients. The distribution of patients based on their preoperative biopsies, and subsequent treatment is illustrated in Fig. 1.

Comparison of patients with a final diagnosis of VSCC $(n=64)$, and without a final diagnosis of VSCC $(n=49)$

On univariate analysis, mean age was significantly higher $(\mathrm{p}=$ 0.03 ), and suspicion of VSCC on the pre-operative biopsy was significantly more frequent $(\mathrm{p}<0.001$ ) for patients with VSCC. No other statistically significant difference was found [Table 1]. On multivariable analysis, only suspicion of VSCC in the pre-operative biopsy remained a significant predictor of a final diagnosis of VSCC $(\mathrm{p}<0.001)$ [Supplementary Table S1].

Forty patients with VSCC had received local treatment, and a second surgery was required for 25 of these patients. Twenty-four patients with VSCC had received radical treatment, and second surgery was required for 3 of these patients [Fig. 1]. Thus, $56 \%$ (36/ 64 ) of patients with VSCC had received definitive treatment, and 44 $\%(28 / 64)$ had received incomplete treatment. Additional surgeries performed for VSCC patients with incomplete treatment included local treatment (WLE) in 6 cases, radical treatment in 11 cases (WLE with SLN procedure in 8 cases, and WLE with LND in 3 cases), and groin treatment in 11 cases (SLN procedure in 8 cases and LND in 3 cases) [Fig. 1].

Forty-five patients without VSCC had received local treatment, which was the definitive treatment, and 4 had received radical treatment (WLE with SLN procedure), which was an over-treatment 
Table 2

Pathology details of VSCC cases $(n=64)$.

\begin{tabular}{ll}
\hline Tumor characteristics & $\begin{array}{l}\text { Number of patients } \\
\text { (percentage) }\end{array}$ \\
\hline I. Depth of invasion & \\
$<1 \mathrm{~mm}$ & $8(13)$ \\
$>1 \mathrm{~mm}$ & $49(77)$ \\
Unknown & $7(10)$ \\
II. Focality & \\
Unifocal & $54(84)$ \\
Multifocal & $10(16)$ \\
III. Differentiation grade & \\
Well & $25(39)$ \\
Moderate & $33(52)$ \\
Poor & $6(9)$ \\
IV. Perineural invasion & \\
Present & $3(5)$ \\
Absent & $61(95)$ \\
V. Lymphovascular space invasion & \\
Present & $2(3)$ \\
Suspicion & $7(11)$ \\
Absent & $55(86)$ \\
VI. Pathology of the groin lymph node $(\mathbf{n}=$ & \\
$\quad$ 24) & \\
Positive & $5(21)$ \\
Negative & $19(79)$ \\
\hline &
\end{tabular}

[Fig. 1]. For 3 of these patients, the final diagnosis on post-operative histopathology was VIN, and for 1 patient, this was lichenoid changes. Thus, treatment administered was definitive for $92 \%$ (45/49), and over-treatment for $8 \%(4 / 49)$ of patients without VSCC.
Comparison of patients with a suspicion of VSCC $(n=55)$, and without a suspicion of VSCC $(n=58)$ in pre-operative biopsy

Suspicion of VSCC was present in the pre-operative biopsy in $49 \%(55 / 113)$ of all included patients. Radical treatment had been performed for $40 \%(22 / 55)$ of patients with a suspicion of VSCC, compared to $10 \%$ (6/58) of patients without a suspicion of $\operatorname{VSCC}(\mathrm{p}<0.001)$ [Table 3]. Radical treatment on patients with a suspicion of VSCC resulted in over-treatment in only 1 case [Fig. 1].

The final diagnosis was VSCC in $82 \%(45 / 55)$ of patients with a suspicion, compared to $33 \%$ (19/58) of patients without a suspicion of VSCC on biopsy ( $\mathrm{p}<0.001$ ). Suspicion of VSCC had a positive predictive value (PPV) of $82 \%$, sensitivity of $70 \%$, and specificity of $80 \%$ for a final diagnosis of VSCC.

\section{Treatment rationale}

Treatment rationale was not clearly documented for every case, hence statistical analyses could not be performed. Information could be retrieved for the patients with a suspicion of VSCC $(n=55)$ on biopsy.

Radical treatment was administered to 22 patients; WLE with SLN procedure for 14 patients, and WLE with LND for 8 patients. Radical treatment was chosen on the grounds of strong clinical suspicion, based on the clinical features. This resulted in definitive treatment of 19 patients, incomplete treatment of 2 patients, and over-treatment of 1 patient.

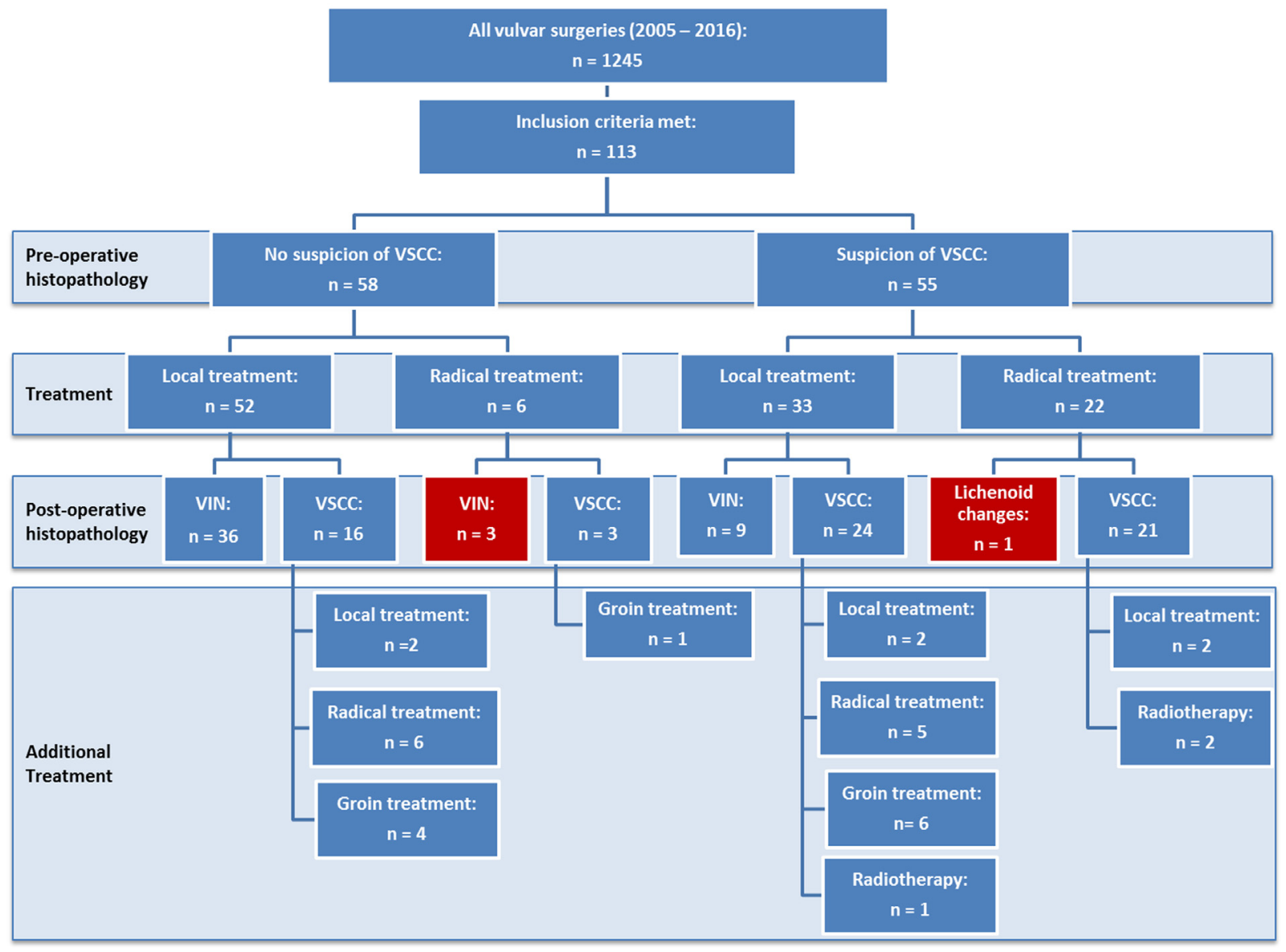

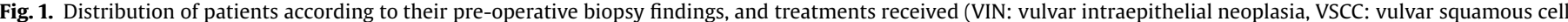

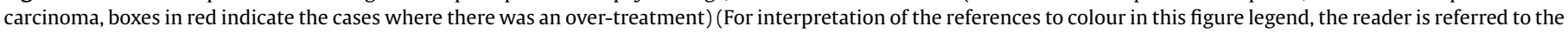
web version of this article). 
Table 3

Characteristics of patients with and without a suspicion of VSCC on pre-operative biopsy.

\begin{tabular}{|c|c|c|c|}
\hline Characteristics & Suspicion of VSCC $(n=55)$ & No suspicion of VSCC $(n=58)$ & p-value* \\
\hline \multicolumn{4}{|l|}{ I. Age (in years) } \\
\hline Mean (95\% Confidence Interval) & $67.9(64.3-71.5)$ & $62.8(58.7-66.9)$ & 0.06 \\
\hline Range & $\begin{array}{l}41-91 \\
\text { Number of patients (percentage) }\end{array}$ & $24-88$ & \\
\hline \multicolumn{3}{|l|}{ II. History of vulvar lesions } & 0.27 \\
\hline None & $36(65)$ & $29(50)$ & \\
\hline Lichenoid lesions & $5(9)$ & $4(7)$ & \\
\hline VIN & $6(11)$ & $11(19)$ & \\
\hline VSCC & $8(15)$ & $14(24)$ & \\
\hline \multicolumn{4}{|l|}{ III. Preoperative clinical examination } \\
\hline \multicolumn{4}{|l|}{ A. Diameter of the lesions } \\
\hline Median (Range) in mm & $30(3-65)$ & $20(3-70)$ & 0.16 \\
\hline$<20 \mathrm{~mm}$ & $20(36)$ & $21(36)$ & 0.62 \\
\hline$\geq 20 \mathrm{~mm}$ & $35(64)$ & $36(62)$ & \\
\hline Unknown & $0(0)$ & $1(2)$ & \\
\hline \multicolumn{4}{|l|}{ B. Focality } \\
\hline Unifocal & $43(78)$ & $44(76)$ & 0.86 \\
\hline Multifocal & $12(22)$ & $14(24)$ & \\
\hline \multicolumn{4}{|l|}{ IV. Surgical procedure } \\
\hline \multicolumn{4}{|l|}{ A. Local treatment } \\
\hline WLE & $33(60)$ & $52(90)$ & \\
\hline B. Radical treatment & & & $<0.001$ \\
\hline WLE + SLN procedure & $14(25)$ & $5(8)$ & \\
\hline WLE + LND & $8(15)$ & $1(2)$ & \\
\hline \multicolumn{3}{|l|}{ V. Post-operative histopathology } & $<0.001$ \\
\hline Lichenoid changes & $1(2)$ & $0(0)$ & \\
\hline VIN & $9(16)$ & $39(67)$ & \\
\hline VSCC & $45(82)$ & $19(33)$ & \\
\hline \multicolumn{3}{|l|}{ VI. Treatment categorization } & 0.55 \\
\hline Definitive & $39(71)$ & $42(73)$ & \\
\hline Incomplete treatment & $15(27)$ & $13(23)$ & \\
\hline Over-treatment & $1(2)$ & $3(4)$ & \\
\hline \multicolumn{3}{|l|}{ VII. Additional treatment } & 0.34 \\
\hline None & $37(66)$ & $45(78)$ & \\
\hline Local treatment (WLE) & $4(7)$ & $2(3)$ & \\
\hline \multicolumn{4}{|c|}{ Radical treatment (WLE + groin treatment) } \\
\hline WLE + SLN & $3(5)$ & $5(8)$ & \\
\hline WLE + LND & $2(4)$ & $1(2)$ & \\
\hline \multicolumn{4}{|l|}{ Groin treatment only } \\
\hline SLN & $3(6)$ & $5(9)$ & \\
\hline LND & $3(6)$ & $0(0)$ & \\
\hline Radiotherapy & $3(6)$ & $0(0)$ & \\
\hline \multicolumn{3}{|l|}{ VIII. Follow up } & 0.58 \\
\hline No evidence of disease & $43(78)$ & $47(81)$ & \\
\hline \multicolumn{4}{|l|}{ Death } \\
\hline Related to disease & $2(4)$ & $1(2)$ & \\
\hline Unrelated to disease & $1(2)$ & $3(4)$ & \\
\hline \multicolumn{4}{|l|}{ Recurrence of disease } \\
\hline VIN & $3(5)$ & $4(7)$ & \\
\hline VSCC & $2(4)$ & $1(2)$ & \\
\hline Under palliative care & $4(7)$ & $1(2)$ & \\
\hline No follow up & $0(0)$ & $1(2)$ & \\
\hline
\end{tabular}

VIN: vulvar intraepithelial neoplasia, VSCC: vulvar squamous cell carcinoma, WLE: wide local excision, SLN: sentinel lymph node, LND: lymph node dissection. Univariate analysis.

Local treatment (WLE) had been performed for 33 patients. For 7 patients the VSCC was recurrent; a previous SLN had been performed for 1 patient, and LND for 2 patients. Other factors that influenced the choice to abstain from groin treatment were co-morbidity, high patient age, low clinical suspicion of VSCC, and suspicion of only superficial VSCC, or a verrucous carcinoma. For 3 patients, WLE was chosen because of the proximity of the tumor to the anus, to minimize the risk of sphincter injury. This resulted in definitive treatment of 20 patients, and incomplete treatment of 13 patients.

\section{Discussion}

VSCC was diagnosed in the excision specimen in $57 \%$ of patients who underwent vulvar excisions in the absence of a conclusive diagnosis of carcinoma on pre-operative biopsy. A final diagnosis of
VSCC appeared to be significantly associated with higher age $(\mathrm{p}=$ 0.03 ), and suspicion of VSCC on pre-operative biopsy ( $p<0.001$ ), on univariate analysis. However, on multivariable analysis, suspicion of VSCC remained the only significant predictor $(\mathrm{p}<$ 0.001).

For patients with a suspicion of VSCC on biopsy $(n=55)$, radical treatment had been performed more frequently, in comparison to patients without a suspicion of VSCC (40\% vs. $10 \%$; p < 0.001 ). Despite the lack of a conclusive diagnosis of VSCC, suspicion of the pathologist probably enabled the surgeon to perform radical treatment more frequently in these patients. Where there was both clinical and histopathologic suspicion of VSCC $(n=22)$, radical treatment (WLE with SLN procedure) resulted in over-treatment in only 1 case [Fig. 1]. For this patient, the final histopathology showed scar tissue with lichenoid changes; this case may have had a small focus of invasion, which was removed at biopsy. Where the 
surgeon had performed a limited excision despite a suspicion of VSCC on biopsy $(\mathrm{n}=33$ ), high patient age, co-morbidities, location of the tumor close to the anus, and history of previous vulvar surgeries were factors which influenced the decision.

In contrast to previous reports, no significant association of lesional size or focality with a final diagnosis of VSCC was found. Preti et al. identified an association of larger sized, and multifocal VIN lesions with occult SCC on univariate analysis in their study [12]. On multivariable analysis however, they discovered tumor size to be the confounder [12]. Raised lesions have also been associated with occult invasive carcinoma [8,9]. Missed diagnoses of carcinoma have been reported to be more frequent for perineal, clitoral, and labial lesions $[8,9,12]$. Similar analyses could not be performed for our cohort as detailed macroscopic descriptions, or the exact anatomical locations of the tumor were not available for all lesions.

For $30 \%$ of patients with VSCC in our cohort, there was no suspicion of carcinoma on pre-operative biopsy. Unsuspected invasion is known to be present in $3.2-22 \%$ of biopsies from high grade VIN $[8,10,13]$. These figures however, may be biased, as biopsies are more commonly performed for clinically suspicious lesions, and conservative / topical treatment modalities are otherwise frequently administered for HSIL. These results may also be influenced by the number of biopsies taken. To avoid missing small invasive foci, thorough sampling of VIN is of undeniable importance.

On histopathology review, the diagnoses did not change for any case. For biopsies reported as suspicious for VSCC, complex anastomosing epithelial architecture, accompanied by inflammatory infiltrate was often observed (results not presented). In a few cases, an overwhelming VIN lesion masked underlying small nests of invasion, which were not sampled in the biopsy. Accurate judgment of invasion can be hindered by tangential sectioning of the biopsy specimen, or in biopsies from the central part of carcinoma, without adjacent 'normal' epithelium, or underlying stroma. Evenly spaced nests with rounded or bulbous contours, without desmoplastic stromal reaction are more likely to be the result of tangential sectioning [14,15]. True invasion, in contrast, is characterized by single cells, or nests of keratinocytes, with irregular or angulated contours, invading from the basilar epidermis or from elongated rete ridges, occasionally accompanied by desmoplastic stromal reaction, edema, or inflammation [14,15].

To assess its adequacy, the surgical treatment administered was categorized as definitive, incomplete, and over-treatment. Seventy-two percent $(81 / 113)$ of patients had received definitive treatment; the final diagnosis was VIN for $56 \%$, and VSCC for 44 $\%$. Twenty-five percent (28/113) of patients had received incomplete treatment; their final diagnosis was VSCC. For these patients, additional local treatment was needed in 6 cases, radical treatment in 11 cases, and only groin treatment in 11 cases [Fig. 1]. There was an over-treatment of 4 patients (3\%); the final diagnosis was VIN for 3 patients, and lichenoid changes for 1 patient.

Our findings reflect the fact that in daily clinical practice, treatment decisions are not based entirely on the pre-operative histopathology, but on the surgeon's practical experience and judgment as well. A surgeon's suspicion for VSCC, needless to say, is not a tangible parameter, and we found that it was not always well documented. As a result, this factor could not be used for the analysis. Nevertheless, through this study, we aimed to enhance our understanding of clinical practice for patients where official guidelines are not directly applicable. Management of patients without a conclusive diagnosis of carcinoma on biopsy is not a well-studied area, and available literature is very limited. The high PPV ( $82 \%$ ) of suspicion of VSCC on pre-operative biopsy, and the fact that the majority ( $72 \%$ ) of patients received definitive treatment, reflects the expertise of both the pathologist and the clinician, and demonstrates the benefits of a referral gynecologic oncology service. However, due to the retrospective nature of the study, some clinical data could not be accessed, and being a singlecenter study, our results may not be generalizable; these are potential limitations.

\section{Conclusion}

Suspicion of VSCC on pre-operative histopathology is a significant predictor of a final diagnosis of VSCC. In our cohort, radical treatment performed on patients with strong clinical and histopathologic suspicion of VSCC helped avoid second surgeries and led to minimal over-treatment. In-depth, well-documented clinical notes on treatment rationale, and protocol deviation (where applicable), along with studying similar multi-institutional cohorts could contribute to the development of improved clinicopathologic algorithms for patient management.

\section{Contributors}

L.W. Jonker: study design, data collection, manuscript writing. S. Dasgupta: statistical analysis, histopathology review, manuscript writing. L.W. Jonker and S. Dasgupta are shared first authors. P.C. Ewing-Graham: histopathology review, scientific input, data discussion, manuscript editing. H.C. van Doorn: study design, scientific input, data discussion, manuscript editing, study supervision.

\section{Funding}

No external funding received.

\section{Patient consent for publication}

Not required.

\section{Ethics approval}

The study was approved by the by the Medical Research Ethics Committee of Erasmus MC Cancer Institute (MEC 2017134).

\section{Data availability statement}

Data are available for sharing in a secure manner, for research and educational purposes, obeying our hospital policies upon reasonable request. Requests can be addressed to Dr. H.C. van Doorn (h.vandoorn@erasmusmc.nl).

\section{Declaration of Competing Interest}

The authors have no conflicts of interest to declare

\section{Acknowledgement}

No external funding was received.

\section{Appendix A. Supplementary data}

Supplementary material related to this article can be found, in the online version, at doi:https://doi.org/10.1016/j.ejogrb.2020.03.027.

\section{References}

[1] Dellinger TH, Hakim AA, Lee SJ, Wakabayashi MT, Morgan RJ, Han ES. Surgical management of vulvar cancer. J Natl Compr Canc Netw 2017;15:121-8, doi: http://dx.doi.org/10.6004/jnccn.2017.0009. 
[2] Lawrie TA, Nordin A, Chakrabarti M, Bryant A, Kaushik S, Pepas L. Medical and surgical interventions for the treatment of usual-type vulval intraepithelial neoplasia. Cochrane Database Syst Rev 2016;1, doi:http://dx.doi.org/10.1002/ 14651858.CD011837.pub2 CD011837.

[3] Tosti G, Iacobone AD, Preti EP, et al. The role of photodynamic therapy in the treatment of vulvar intraepithelial neoplasia. Biomedicines 2018, doi:http:// dx.doi.org/10.3390/biomedicines6010013 pii: E13.

[4] de Witte CJ, van de Sande AJ, van Beekhuizen HJ, Koeneman MM, Kruse AJ, Gerestein CG. Imiquimod in cervical, vaginal and vulvar intraepithelial neoplasia: a review. Gynecol Oncol 2015;139:377-84, doi:http://dx.doi.org/ 10.1016/j.ygyno.2015.08.018.

[5] Oncoline: Dutch National Guidelines. https://www.oncoline.nl/vin. Last accessed Mar, 2020.

[6] Royal College of Obstetricians and Gynecologists: Guidelines for the Diagnosis and Management of Vulval Carcinoma. https://www.rcog.org.uk/globalassets/ documents/guidelines/vulvalcancerguideline.pdf. Last accessed Mar, 2020.

[7] Oncoline: Dutch National Guidelines. https://www.oncoline.nl/vulvacarcinoom. Last accessed Mar, 2020.

[8] Husseinzadeh N, Recinto C. Frequency of invasive cancer in surgically excised vulvar lesions with intraepithelial neoplasia (VIN 3). Gynecol Oncol 1999;73:119-20, doi:http://dx.doi.org/10.1006/gyno.1998.5327.

[9] Chafe W, Richards A, Morgan L, Wilkinson E. Unrecognized invasive carcinoma in vulvar intraepithelial neoplasia (VIN). Gynecol Oncol 1988;31:154-60, doi: http://dx.doi.org/10.1016/0090-8258(88)90284-3.
[10] Modesitt SC, Waters AB, Walton L, Fowler Jr. WC, Van Le L. Vulvar intraepithelial neoplasia III: occult cancer and the impact of margin status on recurrence. Obstet Gynecol 1998;92:962-6, doi:http://dx.doi.org/10.1016/ s0029-7844(98)00350-0.

[11] Polterauer S, Dressler AC, Grimm C, et al. Accuracy of preoperative vulva biopsy and the outcome of surgery in vulvar intraepithelial neoplasia 2 and 3 . Int J Gynecol Pathol 2009;6:559-62, doi:http://dx.doi.org/10.1097/ PGP.0b013e3181a934d4.

[12] Preti M, Bucchi L, Ghiringhello B, et al. Risk factors for unrecognized invasive carcinoma in patients with vulvar high grade squamous intraepithelial lesion at vulvoscopy-directed biopsy. J Gynecol Oncol 2017;28:e27, doi:http://dx.doi. org/10.3802/jgo.2017.28.e27.

[13] van Seters M, van Beurden M, de Craen AJ. Is the assumed natural history of vulvar intraepithelial neoplasia III based on enough evidence? A systematic review of 3322 published patients. Gynecol Oncol 2005;97:645-51, doi:http:// dx.doi.org/10.1016/j.ygyno.2005.02.012.

[14] del Pino M, Rodriguez-Carunchio L, Ordi J. Pathways of vulvar intraepithelial neoplasia and squamous cell carcinoma. Histopathology 2013;62:161-75, doi: http://dx.doi.org/10.1111/his.12034.

[15] Yang EJ, Kong CS, Longacre TA. Vulvar and anal intraepithelial neoplasia: terminology, diagnosis, and ancillary studies. Adv Anat Pathol 2017;3:136-50, doi:http://dx.doi.org/10.1097/PAP.0000000000000149. 